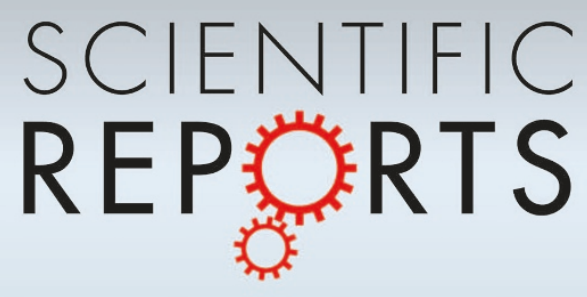

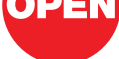

SUBJECT AREAS: SPINTRONICS

MOLECULAR ELECTRONICS

ELECTRONIC PROPERTIES AND MATERIALS

MAGNETIC PROPERTIES AND MATERIALS

Received

4 December 2012

Accepted

3 January 2013

Published

4 February 2013

Correspondence and requests for materials should be addressed to H.J.G. (higao@iphy. ac.cn)

$\dagger$ These authors contributed equally to this work.

\section{Reversible Single Spin Control of Individual Magnetic Molecule by Hydrogen Atom Adsorption}

\author{
Liwei Liu' ${ }^{1 \dagger}$, Kai Yang ${ }^{1 \dagger}$, Yuhang Jiang ${ }^{1 \dagger}$, Boqun Song', Wende Xiao', Linfei Li', \\ Haitao Zhou', Yeliang Wang', Shixuan Du' , Min Ouyang ${ }^{2}$, Werner A. Hofer ${ }^{1,3}$, \\ Antonio H. Castro Neto ${ }^{4} \& \mathrm{Hong}^{-J u n} \mathrm{Gao}^{1}$
}

${ }^{1}$ Institute of Physics, Chinese Academy of Sciences, P.O. Box 603, Beijing 100190, China, ${ }^{2}$ Department of Physics and Center for Nanophysics and Advanced Materials, University of Maryland, College Park, MD 20742, USA, ${ }^{3}$ Department of Physics, The University of Liverpool, Liverpool L69 3BX, UK, ${ }^{4}$ Graphene Research Centre, Department of Physics, National University of Singapore, 117542, Singapore.

The reversible control of a single spin of an atom or a molecule is of great interest in Kondo physics and a potential application in spin based electronics. Here we demonstrate that the Kondo resonance of manganese phthalocyanine molecules on a $\mathrm{Au}(111)$ substrate have been reversibly switched off and on via a robust route through attachment and detachment of single hydrogen atom to the magnetic core of the molecule. As further revealed by density functional theory calculations, even though the total number of electrons of the $\mathrm{Mn}$ ion remains almost the same in the process, gaining one single hydrogen atom leads to redistribution of charges within $3 d$ orbitals with a reduction of the molecular spin state from $S=3 / 2$ to $S=1$ that directly contributes to the Kondo resonance disappearance. This process is reversed by a local voltage pulse or thermal annealing to desorb the hydrogen atom.

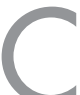

ontrol over charge and spin states at the single molecule level is crucial not only for a fundamental understanding of charge and spin interactions but also represents a prerequisite for development of molecular electronics and spintronics ${ }^{1-3}$. While charge manipulation has been demonstrated by gas adsorption $^{4}$ and atomic manipulation ${ }^{5}$, the reversible control of a single spin of an atom or a molecule has been challenging. Typically, atomic or molecular spin can be probed as a Kondo effect that manifests itself as a conductance anomaly at the Fermi level when it is coupled to a metallic system ${ }^{6,7}$. However, an effective method to manipulate molecular spin both individually and ensemble has been limited ${ }^{8}$. In this paper, we present the reversible control of a single spin in manganese phthalocyanine molecules on a $\mathrm{Au}(111)$ substrate. This process can be reversed by a local voltage pulse or thermal annealing to desorb the hydrogen atom, accompanied by a recovery of the molecular Kondo resonance.

Molecules with a single metal ion, including metal phthalocyanines (MPcs), metal porphyrins (MPs) and their derivatives, are ideal prototypes to study spin related phenomena due to their versatility and the tunability of charge and spin properties ${ }^{9,10}$. When magnetic MPcs or MPs are placed on metal surfaces, the Kondo resonance induced by the interaction between a localized spin of the molecule and conduction electrons from the substrate manifests itself as a narrow, pronounced peak in the density of states close to the Fermi level. While many attempts have been applied to govern the Kondo process, including conformation variation ${ }^{11-15}$, alteration of adsorption site $^{16,17}$, quantum size effects ${ }^{18}$, ligand attachment ${ }^{19}$, molecular assembly ${ }^{20}$ and atomic doping ${ }^{21}$, most are either irreversible or cannot manipulate the Kondo effect at both single-molecule scale and ensemble; an effective method of molecular spin manipulation has been limited. We show that spin state of a single manganese phthalocyanine ( $\mathrm{MnPc})$ molecule on the $\mathrm{Au}(111)$ can be reversibly switched by chemical absorption and desorption of a single hydrogen atom. Our novel reversible spin control scheme can be easily realized at both ensemble of molecules and single-molecule level, which opens up new avenue of broader applications based on molecular spin state.

\section{Results}

Figure 1a shows a typical low temperature scanning tunneling microscopy (LT-STM) image of MnPc molecules on an $\mathrm{Au}(111)$ substrate, in which every $\mathrm{MnPc}$ molecule possesses three inherent unpaired electrons with a total 
a

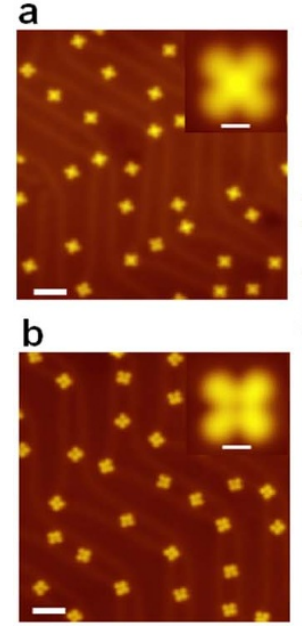

C

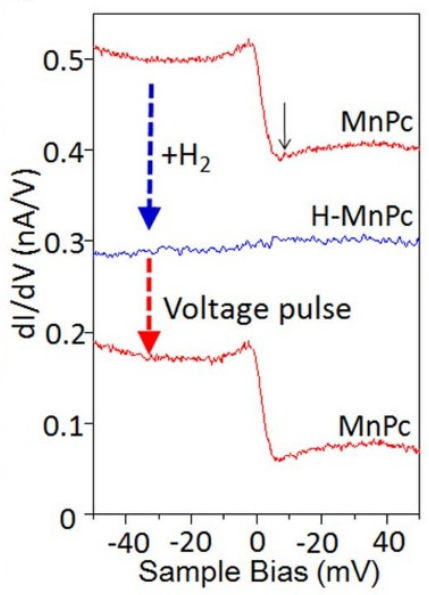

d

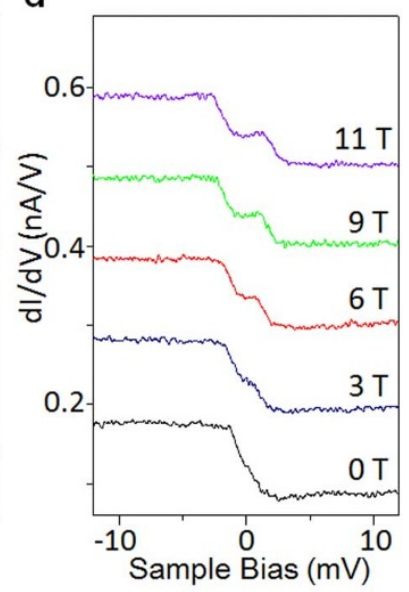

Figure $1 \mid$ Topography and $d I / d V$ curves of the MnPc molecules on the Au(111) before and after hydrogen atom adsorption. (a) Topography of the $\mathrm{MnPc}$ molecules on the $\mathrm{Au}(111)$ before hydrogen addition, showing molecules with bright center. Scanning bias: - $0.2 \mathrm{~V}$; Tunneling current: 10 pA; Scale bar, $5 \mathrm{~nm}$. (Inset) High-resolution image of a single molecule. Scale bar, $0.7 \mathrm{~nm}$. (b) Topography of the MnPc molecules after hydrogen atom decoration, showing some molecules with suppressed center. Scanning bias: $-0.2 \mathrm{~V}$; Tunneling current: $10 \mathrm{pA}$; Scale bar, $5 \mathrm{~nm}$. (Inset) High-resolution image of a single molecule with suppressed center. Scale bar, $0.7 \mathrm{~nm}$. (c) Sequential variation of the $d I / d V$ spectra recorded at the center of a MnPc molecule induced by the absorption and desorption of a single hydrogen atom. For comparison purpose spectra were vertically shifted $0.1 \mathrm{nA} / \mathrm{V}$ and $0.33 \mathrm{nA} / \mathrm{V}$ for middle and upper curves, respectively. (d) $d I / d V$ spectra acquired at the center of the MnPc molecule under external magnetic field at $0.4 \mathrm{~K}$, showing the splitting of Kondo resonance. For clarity the successive spectra were vertically shifted by $0.1 \mathrm{nA} / \mathrm{V}$.

spin of $S=3 / 2$. After deposition of 0.05 monolayer of MnPc onto a $22 \times \sqrt{3}$ herringbone reconstructed $\mathrm{Au}(111)$ surface, isolated $\mathrm{MnPc}$ molecules preferentially occupy the elbow and face centered cubic (fcc) sites. The STM image unambiguously confirms an intact molecular structure after thermal deposition with exhibition of a protruding "cross" feature with four-fold symmetry ${ }^{16,18}$. After the $\mathrm{MnPc} / \mathrm{Au}(111)$ sample was dosed with 30 Langmuir (L) of $\mathrm{H}_{2}$ at room temperature (RT) and subsequently cooled down to $4.2 \mathrm{~K}$, all molecules retained the cross feature but some showed a depression at the center. After extensive $\mathrm{H}_{2}$ dosage, all of the original MnPc molecules can be turned into molecules with a depression at the center (Fig. 1b and Supplementary Fig. 1).

The variation of the molecular morphology after the $\mathrm{H}_{2}$ gas dosage can be attributed to the chemical adsorption of a single hydrogen atom onto the MnPc molecule, according to key control experiments summarized as follows: (1) This change of molecular morphology is reversible. We observed that by applying a positive voltage pulse (e.g. $2.0 \mathrm{~V}$ for one second with feedback loop off) the bright protrusion feature of the $\mathrm{MnPc}$ molecules can be recovered on a depression. This behavior is consistent with a tip induced detachment ${ }^{4}$ of adsorbates from the MnPc molecule. Alternatively, the depression feature can be switched back to a bright protrusion by thermal annealing of samples at $500 \mathrm{~K}$ for 10 mins. (2) The apparent height of the molecule remains unchanged when the $\mathrm{H}_{2}$ is dosed at $4.2 \mathrm{~K}$ instead of RT, even with very high dosage capacity $(1000 \mathrm{~L})$. We observed that intact $\mathrm{H}_{2}$ molecules can only reside around the MnPc molecules without modification of the intrinsic molecular structure (Supplementary Fig. 2). This suggests that the interaction between the $\mathrm{H}_{2}$ and the MnPc molecules is weak and absorption of molecular hydrogen cannot be the origin of the process demonstrated in the Fig. 1. (3) A threshold voltage of $+1.28 \mathrm{~V}$ is required in order to detach the adsorbate from the host MnPc molecule (Supplementary Fig. 3). This threshold voltage suggests the binding between adsorbate and the molecule is chemical adsorption rather than physical adsorption, which demonstrates that the absorption of the $\mathrm{H}_{2}$ cannot be the origin of the process shown in the Fig. 1. (4) Lastly we also carried out one control experiment by direct dosage of atomic hydrogen $\operatorname{gas}^{22}$ (Supplementary Section 2.), and similar features of the molecular morphology change were also obtained. From these key experiments we conclude that the depression feature after dosage of hydrogen gas is due to adsorption of atomic hydrogen (labeled as "H-MnPc").

We explored and compared in detail the difference of electronic structure between the MnPc and the $\mathrm{H}-\mathrm{MnPc}$ states by measuring differential conductance $(d I / d V)$ spectra. As shown in the upper red curve in Fig. 1c, the $d I / d V$ spectra acquired at the center of the original MnPc molecules consistently show a pronounced step shaped feature at zero bias, regardless of the molecular adsorption configurations and locations on the Au terraces. Such step shaped feature in $d I / d V$ spectra is very sharp and close to the Fermi level. This spectra feature manifests linear splitting at the presence of magnetic field (Fig. 1d) as well as temperature dependence (Supplementary Fig. 8), and thus it can be safely attributed to the Kondo resonance ${ }^{6,7,23,24}$. In contrast, the $d I / d V$ spectra of the $\mathrm{H}-\mathrm{MnPc}$ state (middle blue curve in Fig. 1c) are featureless in this energy range. Importantly, once the $\mathrm{H}-\mathrm{MnPc}$ state was switched back to the MnPc state, its electronic structure including the Kondo resonance can be fully recovered (bottom red curve in Fig. 1c) in addition to the recovery of the topographic feature.

Further insight into the reversible switching of the Kondo resonance between the $\mathrm{MnPc}$ and the $\mathrm{H}-\mathrm{MnPc}$ states can be gained by density functional theory (DFT) calculations (see Method for more details). Topographic features of both the $\mathrm{MnPc}$ and $\mathrm{H}$ MnPc states can be well reproduced by STM simulations by integrating electron density from $-0.4 \mathrm{eV}$ to the Fermi level according to the Tersoff-Hamann approximation ${ }^{25}$. The projected density of states (PDOS) shows that the bright protrusion at the molecular center of MnPc is due to the $d$-orbital character of the Mn ion near the Fermi level (Fig. 2a, and Supplementary Fig. 5). The calculated binding energy of $1.75 \mathrm{eV}$ between the hydrogen atom and $\mathrm{MnPc} /$ $\mathrm{Au}(111)$ indicates that $\mathrm{H}$ on $\mathrm{MnPc} / \mathrm{Au}(111)$ is of a chemical adsorption with binding energy in the same range of our experimental value. Therefore, both experimental results and theoretical calculations suggest that attaching and detaching a single hydrogen atom from the central $\mathrm{Mn}$ ion is the physical origin of spin switching process. 

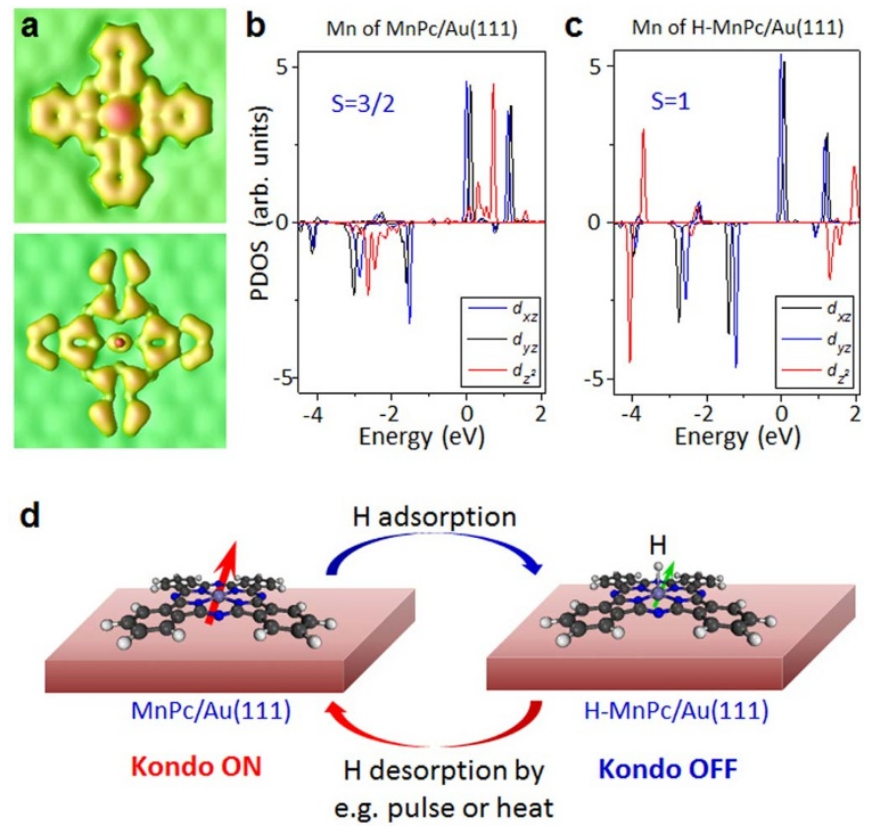

Figure $2 \mid$ STM simulation and PDOS of the MnPc/Au(111) before and after hydrogen adsorption (a) STM simulation of the $\mathrm{MnPc} / \mathrm{Au}(111)$ and the $\mathrm{H}-\mathrm{MnPc} / \mathrm{Au}(111)$, confirming the topographic feature in Fig. 1a and b. (b) and (c) PDOS of $\mathrm{d}_{\mathrm{xz}}, \mathrm{d}_{\mathrm{yz}}$ and $d_{z^{2}}$ orbitals of Mn ion in the MnPc/ $\mathrm{Au}(111)$ and the $\mathrm{H}-\mathrm{MnPc} / \mathrm{Au}(111)$, respectively, revealing that spin at the $\mathrm{Mn}$ ion is reduced from 3/2 to 1 . (d) Schematics of reversible control of molecular Kondo effect with adsorption (Kondo OFF) and desorption of hydrogen atom (Kondo ON).

\section{Discussions}

An analysis of the electronic structure of $\mathrm{MnPc}$ and $\mathrm{H}-\mathrm{MnPc}$ reveals that the number of $d$-orbital electrons of the $\mathrm{Mn}$ ion remains almost unchanged. Thus the additional electron contributed by the hydrogen atom is donated to the Pc skeleton. However, the effective charges in individual $d$ orbitals are redistributed. Before hydrogen adsorption, the Mn ion in the MnPc molecule has a net spin of $\mathrm{S}=$ $3 / 2$. This is due to two partially paired orbitals, $d_{x z}$ and $d_{y z}$, and two unpaired orbitals, $d_{x y}$ and $d_{z^{2}}$ (Fig. 2b). The Kondo resonance observed in the $d I / d V$ spectra in this case originates from an exchange interaction between the localized spin of the magnetic $\mathrm{Mn}$ ion and the conducting electrons of the $\mathrm{Au}(111)$ substrate $^{26}$. After atomic hydrogen decoration (i.e., in the H-MnPc state), the spin polarization decreases in the $d_{z^{2}}$ orbital: Before adsorption the spin-up charge in this state is close to one while the spin-down charge is close to zero. After adsorption the charge in both spin states is close to $1 / 2$. This leads to a decrease of the net spin of the $\mathrm{Mn}$ ion from $\mathrm{S}=$ $3 / 2$ to $S=1$ (Fig. 2c). Because the Kondo effect strongly depends on the coupling between the localized spin and the conducting electrons, a reduced molecular spin state directly contributes to the suppression of the Kondo effect (Supplementary Section 4) ${ }^{27,28}$. A schematics of manipulation of the molecular Kondo effect by hydrogen adsorption and desorption is illustrated in Fig. 2d.

In principle, the appearance or disappearance of the Kondo resonance on this magnetic molecule can be seen as a single bit of information. This may have implications for information recording and storage at the ultimate molecular limit ${ }^{10-12,29-31}$, when combined with STM capability of atomic manipulation of molecular adsorption and desorption. We explore this avenue by investigating an ordered molecular array. Fig. 3a shows topography and $d I / d V$ mapping acquired simultaneously from a $3 \times 4$ molecular array. All the $\mathrm{MnPc}$ molecules within the array are initialized by converting them to the $\mathrm{H}-\mathrm{MnPc}$ state through the adsorption of hydrogen atoms, with evidence of featureless $d I / d V$ mapping (i.e. absence of the Kondo resonance), while the four-lobe topographic structure of individual molecules remains (Fig. 3a). By applying voltage pulses on selected $\mathrm{H}-\mathrm{MnPc}$ molecules, we can precisely address an individual molecule within the array and the $\mathrm{H}-\mathrm{MnPc}$ molecules can be converted back to the MnPc state one-by-one. As a result, a pattern of confined dark dot feature corresponding to the Kondo resonance of the MnPc molecules can be created in the $d I / d V$ mapping (Fig. 3b). Importantly, this process can be extended to an ultimate close-packed array with the highest molecular density (Supplementary Fig. 6 and Supplementary Fig. 7), with one example shown in the Fig. 3c. While the intermolecular spacing is as small as $1.4 \mathrm{~nm}$ in a close-packed array, all the dark-dot features in the $d I / d V$ mapping remain localized at the center of the MnPc molecules, and the $d I / d V$ spectra of the Kondo switching recorded in the molecular assembly show no difference from our observation of isolated molecules (Supplementary Fig. 7), regarding characteristic parameters such as the Fano factor $(q)$ and the Kondo temperature $\left(T_{K}\right)$. Correspondingly, no change occurs in the molecular framework during the spin manipulations within the molecular assembly, in contrast to previously investigated systems, which involve conformational changes at the periphery of the molecule $^{11,13,14}$. A storage system based on the molecular spin states thus describes the ultimate limit of information storage. Furthermore, all the MnPc molecules in the arrays exhibit consistent Kondo features, suggesting a site-independent Kondo resonance and the inter-particle interactions can be weak enough to become negligible in the process. (Fig. 1 and Supplementary Fig. 7). Even more importantly, such molecular spin switching can be consistently achieved backand-forth for many times with no observable change of the Kondo features. To substantiate the robustness of the spin switching process, we have quantified both $T_{K}$ and $q$ of MnPc state from a Fano fitting (Supplementary Fig. 8) and monitored both parameters during the spin switching process by repeatedly absorption and desorption of $\mathrm{H}$-atom, with results summarized in the Fig. $3 \mathrm{~d}$. That both, the $T_{K}$ and $q$ values in the spin manipulation cycles remain constant supports the robustness of this process. Atomic localization feature of the Kondo resonance switching makes it possible to create more complex patterns based on molecular spin state, including one example of a digitalization from (000) to (111) that can be fabricated from a larger $3 \times 8$ molecular array (Supplementary Fig. 9).

The ability to reversibly tune the Kondo effect at the molecule level by gaining or losing a single hydrogen atom creates a new class of simplest molecular Kondo system in which the spin screen can be manipulated directly through the control of the local atomic environment. Our demonstration of reversible molecular spin control by chemical stimuli is different from a few recent works in that it offers an in-situ single molecule characterization and control instead of ensemble measurement ${ }^{32}$ and selection of noble metallic Au substrate can further avoid complex spin texture of substrate due to Rashba type spin-split pair surface states of a semimetal of such as $\mathrm{Bi}^{33,34}$. Even more importantly, our scheme can allow not only ensemble spin manipulation (such as Kondo ON/OFF switching of all molecules by thermal annealing and $\mathrm{H}_{2}$ gas dosage, respectively) but also control at the single-molecule level by atomic manipulation of single $\mathrm{H}$ atom that has not been achieved so $\mathrm{far}^{21}$. It thus opens up a variety of avenues. Our approach can be readily extended to more complex magnetic molecules. The atomically precise engineering of molecular spin states provides a unique test ground for probing magnetic interactions at the single-molecule level. For example, the magnetism in transition-metal ions based magnetic molecules arises from unpaired spins residing in the $d$-orbitals. Controlled coupling of its net spins with another unpaired spin of a well-defined hydrogen atom provides the simplest conceivable systems potentially exhibiting quantum critical behavior. Our demonstration of robust Kondo switching in a molecular array could also be applied as nonvolatile classical information recording and storage at the ultimate molecule limit without the requirement of molecular conformation change. 
a
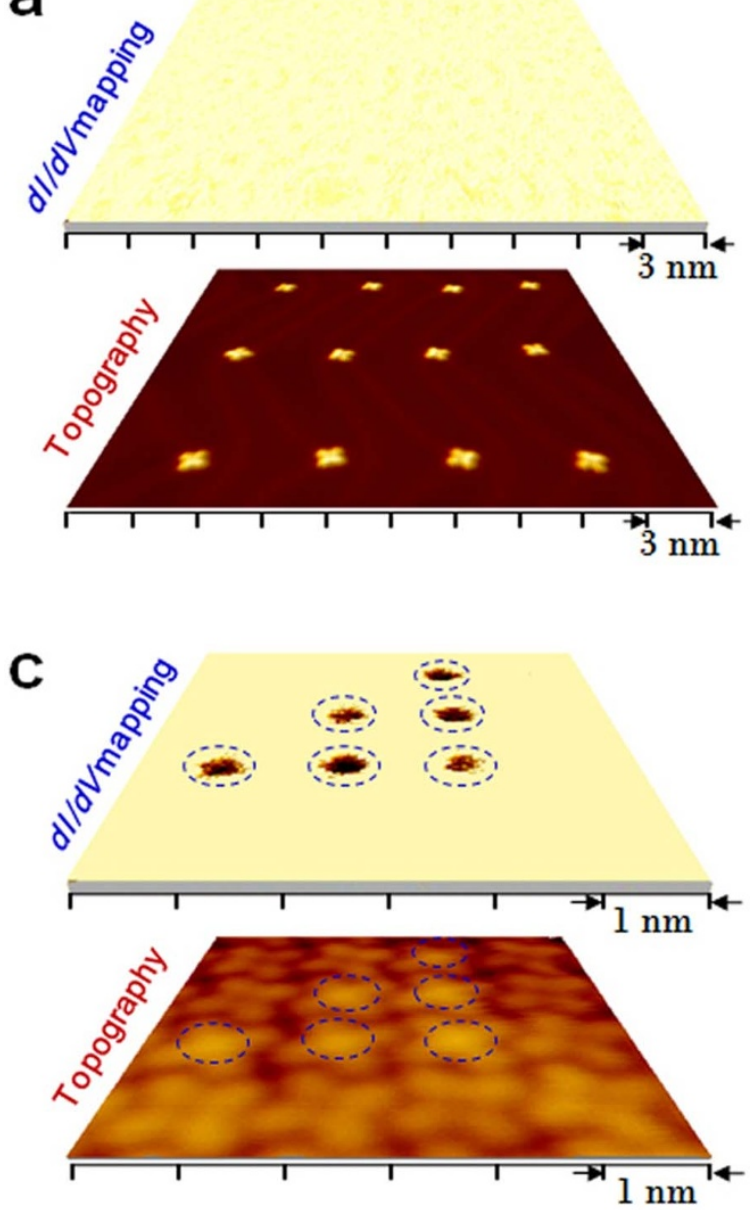

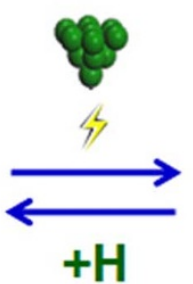

b

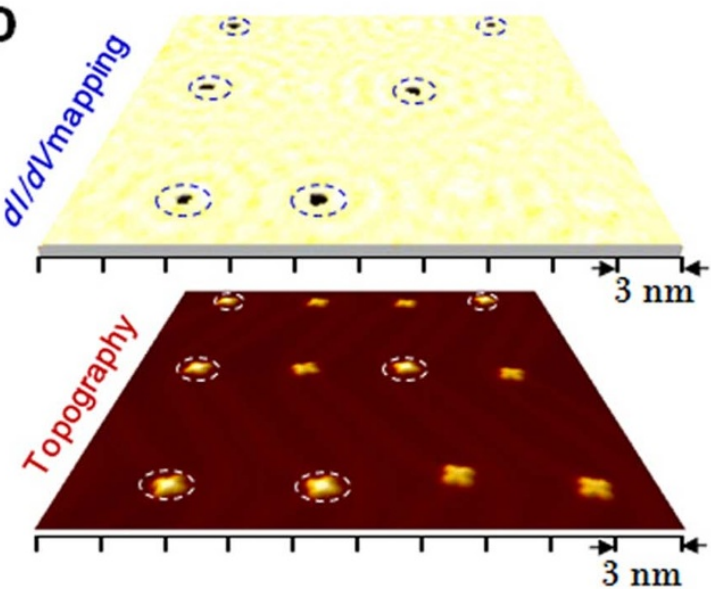

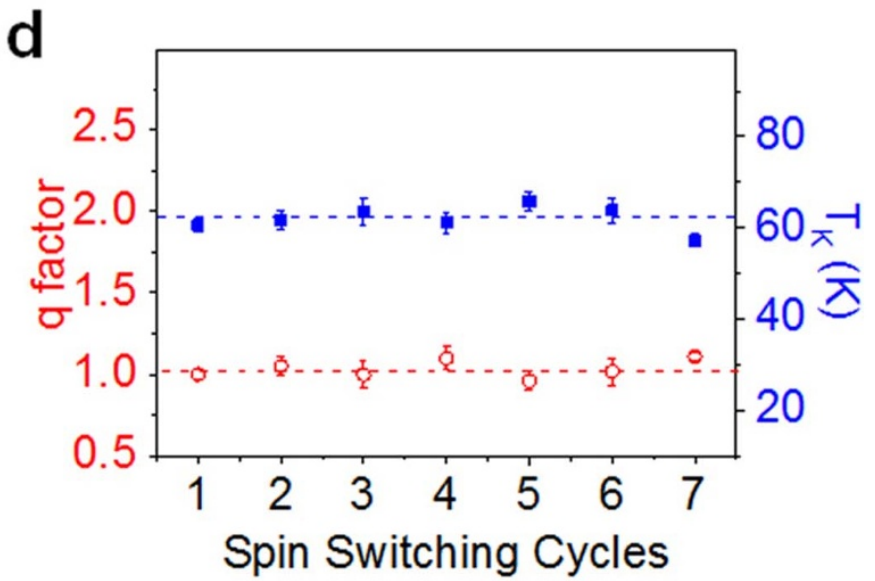

Figure $3 \mid$ Reversible spin switching in molecular arrays. (a) The $d I / d V$ mapping (upper panel) and simultaneously acquired topography (lower panel) of a $\mathrm{H}-\mathrm{MnPc}$ molecular array (Kondo OFF state). (b) The $d I / d V$ mapping (upper panel) and simultaneously acquired topography (lower panel) of the same molecular array as a but with a few selected molecules converted to the MnPc state (Kondo ON). For both (a) and (b), the $d I / d V$ mappings were taken at $6 \mathrm{mV}$. Note that the MnPc state (Kondo ON) can be erased and converted back to H-MnPc state (Kondo OFF) by adsorption of additional hydrogen. (c) The $d I / d V$ mapping (upper panel) and topography (lower panel) of a close-packed molecular array with pre-designed Kondo pattern by switching selected molecules from the H-MnPc to the MnPc states within the array. The $d I / d V$ mapping was taken at $6 \mathrm{mV}$. (d) The Fano factor $q$ and Kondo temperature $T_{K}$ of Kondo ON states after multiple-cycles of spin switching, indicating the robustness of the spin manipulation process.

Future design of more complex molecular framework might offer a way to enable and tailor coherent spin coupling through such as inter-molecular bridge ${ }^{35}$.

\section{Methods}

Experimental method. Experiments were carried out in an ultrahigh vacuum (base pressure of $1 \times 10^{-10} \mathrm{mbar}$ ) LT-STM system (Unisoku), equipped with standard surface processing facilities. An atomically flat $\mathrm{Au}(111)$ surface was prepared by repeated cycles of sputtering with argon ions and annealing at $800 \mathrm{~K}$. Commercial $\mathrm{MnPc}$ molecules (Sigma-Aldrich, $97 \%$ purity) were sublimated from a Knudsen-type evaporator after thermal purification, while the $\mathrm{Au}(111)$ substrate was held at room temperature. One monolayer refers to a close-packed MnPc layer covering the whole $\mathrm{Au}(111)$ surface, as examined by STM (Supplementary Fig. 6). STM images were acquired in the constant-current mode with bias voltage applied to the sample. Spectroscopic measurements were performed by using a lock-in technique with a $0.5 \mathrm{mV}_{\mathrm{rms}}$ sinusoidal modulation at a frequency of $973 \mathrm{~Hz}$. Most images and spectra were obtained with electrochemically etched tungsten tips at $4.2 \mathrm{~K}$, but the magnetic field dependent measurement was performed at $0.4 \mathrm{~K}$. Measurements of local spectroscopy were electronically calibrated by performing $d I / d V$ measurements on clean $\mathrm{Au}(111)$ before and after measurements on molecules, based on the well-known $\mathrm{Au}(111)$ surface state ${ }^{36}$. The atomic $\mathrm{H}$ is generated by cracking $\mathrm{H}_{2}$ molecules with hot tungsten filament, which is placed very close $(\sim 5 \mathrm{~cm})$ to the sample.

Calculation details. The DFT calculations were performed using Vienna ab-initio simulation package (VASP) ${ }^{37}$. Perdew-Wang 91 exchange correlation functionals and the projector augmented-wave method were employed ${ }^{38}$. The energy cutoff for plane-wave basis set was $400 \mathrm{eV}$. A $c(7 \times 8)$ supercell containing three layers of gold atoms was employed to model an isolated molecule on $\mathrm{Au}(111)$ substrate. To ensure that interactions between the periodic slabs through vacuum can be negligible, the slabs were separated by a vacuum gap of $20 \AA$. For geometry optimization, the bottom layer was fixed, while the adsorbates and the other metal layers were allowed to relax until the forces applied to the relaxed atoms were less than $0.01 \mathrm{eV} / \AA$. A single $\Gamma$ point was used in sampling the Brillouin zone due to the numerical limitations (See Supplementary Section 8).

1. Joachim, C., Gimzewski, J. K. \& Aviram, A. Electronics using hybrid-molecular and mono-molecular devices. Nature 408, 541-548 (2000).

2. Moth-Poulsen, K. \& Bjornholm, T. Molecular electronics with single molecules in solid-state devices. Nat. Nanotechnol. 4, 551-556 (2009).

3. Bogani, L. \& Wernsdorfer, W. Molecular spintronics using single-molecule magnets. Nat. Mater. 7, 179-186 (2008).

4. Katano, S., Kim, Y., Hori, M., Trenary, M. \& Kawai, M. Reversible Control of Hydrogenation of a Single Molecule. Science 316, 1883-1886 (2007).

5. Lee, H. J. \& Ho, W. Single-bond formation and characterization with a scanning tunneling microscope. Science 286, 1719-1722 (1999).

6. Li, J. T., Schneider, W. D., Berndt, R. \& Delley, B. Kondo scattering observed at a single magnetic impurity. Phys. Rev. Lett. 80, 2893-2896 (1998).

7. Madhavan, V., Chen, W., Jamneala, T., Crommie, M. F. \& Wingreen, N. S. Tunneling into a single magnetic atom: Spectroscopic evidence of the Kondo resonance. Science 280, 567-569 (1998).

8. Ham, U. \& Ho, W. Spin Splitting Unconstrained by Electron Pairing: The Spin-Vibronic States. Phys. Rev. Lett. 108, 106803 (2012).

9. Atodiresei, N. et al. Design of the Local Spin Polarization at the OrganicFerromagnetic Interface. Phys. Rev. Lett. 105, 066601 (2010). 
10. Wang, Y. F., Kroger, J., Berndt, R. \& Hofer, W. A. Pushing and Pulling a Sn Ion through an Adsorbed Phthalocyanine Molecule. J. Am. Chem. Soc. 131, 3639-3643 (2009).

11. Iancu, V., Deshpande, A. \& Hla, S.-W. Manipulating Kondo Temperature via Single Molecule Switching. Nano Lett. 6, 820-823 (2006).

12. Komeda, T. et al. Observation and electric current control of a local spin in a single-molecule magnet. Nat. Commun. 2, 217 (2011).

13. Miyamachi, T. et al. Robust spin crossover and memristance across a single molecule. Nat. Commun. 3, 938 (2012).

14. Zhao, A. D. et al. Controlling the Kondo Effect of an Adsorbed Magnetic Ion Through Its Chemical Bonding. Science 309, 1542-1544 (2005).

15. Choi, T. et al. A Single Molecule Kondo Switch: Multistability of Tetracyanoethylene on Cu(111). Nano Lett. 10, 4175-4180 (2010).

16. Franke, K. J., Schulze, G. \& Pascual, J. I. Competition of Superconducting Phenomena and Kondo Screening at the Nanoscale. Science 332, 940-944 (2011).

17. Jiang, Y., Zhang, Y. N., Cao, J. X., Wu, R. Q. \& Ho, W. Real-Space Imaging of Kondo Screening in a Two-Dimensional O-2 Lattice. Science 333, 324-328 (2011).

18. Fu, Y. S. et al. Manipulating the Kondo resonance through quantum size effects. Phys. Rev. Lett. 99, 256601 (2007).

19. Wahl, P. et al. Kondo Effect of Molecular Complexes at Surfaces: Ligand Control of the Local Spin Coupling. Phys. Rev. Lett. 95, 166601 (2005).

20. Iancu, V., Deshpande, A. \& Hla, S. W. Manipulation of the Kondo effect via two-dimensional molecular assembly. Phys. Rev. Lett. 97, 266603 (2006).

21. Robles, R. et al. Spin Doping of Individual Molecules by Using Single-Atom Manipulation. Nano Lett. 12, 3609-3612 (2012).

22. Stobinski, L. \& Dus, R. Molecular-Hydrogen Chemisorption on Thin Unsintered Gold-Films Deposited at Low-Temperature. Surf. Sci. 298, 101-106 (1993).

23. Fano, U. Effects of Configuration Interaction on Intensities and Phase Shifts. Phys Rev 124, 1866 (1961)

24. Manoharan, H. C., Lutz, C. P. \& Eigler, D. M. Quantum mirages formed by coherent projection of electronic structure. Nature 403, 512-515 (2000).

25. Tersoff, J. \& Hamann, D. R. Theory and Application for the Scanning Tunneling Microscope. Phys. Rev. Lett. 50, 1998-2001 (1983).

26. Gao, L. et al. Site-specific Kondo effect at ambient temperatures in iron-based molecules. Phys. Rev. Lett. 99, 106402 (2007).

27. Posazhennikova, A., Bayani, B. \& Coleman, P. Conductance of a spin-1 quantum dot: The two-stage Kondo effect. Phys. Rev. B 75, 245329 (2007).

28. Otte, A. F. et al. The role of magnetic anisotropy in the Kondo effect. Nat. Phys. 4 847-850 (2008).

29. Nakaya, M., Tsukamoto, S., Kuwahara, Y., Aono, M. \& Nakayama, T. Molecular Scale Control of Unbound and Bound C-60 for Topochemical Ultradense Data Storage in an Ultrathin C-60 Film. Adv. Mater. (Weinheim, Ger.) 22, 1622 (2010).

30. Donhauser, Z. J. et al. Conductance switching in single molecules through conformational changes. Science 292, 2303-2307 (2001).

31. Liljeroth, P., Repp, J. \& Meyer, G. Current-induced hydrogen tautomerization and conductance switching of naphthalocyanine molecules. Science 317, 1203-1206 (2007).
32. Wäckerlin, C. et al. Controlling spins in adsorbed molecules by a chemical switch. Nat. Commun. 1, 61-67 (2010).

33. Stróżecka, A., Soriano, M., Pascual, J. I. \& Palacios, J. J. Reversible Change of the Spin State in a Manganese Phthalocyanine by Coordination of CO Molecule. Phys. Rev. Lett. 109, 147202 (2012).

34. Pascual, J. I. et al. Role of spin in quasiparticle interference. Phys. Rev. Lett. 93, 196802 (2004).

35. Ouyang, M. \& Awschalom, D. D. Coherent spin transfer between molecularly bridged quantum dots. Science 301, 1074-1078 (2003).

36. Chen, W., Madhavan, V., Jamneala, T. \& Crommie, M. F. Scanning tunneling microscopy observation of an electronic superlattice at the surface of clean gold Phys. Rev. Lett. 80, 1469-1472 (1998).

37. Kresse, G. Ab-Initio Molecular-Dynamics for Liquid-Metals. Phys. Rev. B 47, 558 (1995).

38. Kresse, G. \& Joubert, D. From ultrasoft pseudopotentials to the projector augmented-wave method. Phys. Rev. B 59, 1758-1775 (1999).

\section{Acknowledgements}

The authors would like to thank Wilson Ho for helpful discussion. G. Li, L. Meng, L.H. Yan, and X.M. Fei for experimental assistance. Work at IOP was supported by grants from National Natural Science Foundation of China (grant 10834011, 60921092), National "973" projects of China (grant 2009CB929103, 2011CB921702), the Chinese Academy of Sciences, and SSC. Work in Liverpool is supported by the EPSRC Car-Parinello consortium, grant No EP/F037783/1. M.O. acknowledged support from the ONR

(award \#: N000141110080). AHCN acknowledges DOE grant DE-FG02-08ER46512, ONR grant MURI N00014-09-1-1063, and the NRF award R-144-000-295-281.

\section{Author contributions}

L.W.L., K.Y. and Y.H.J. contribute equally to this work. L.W.L., K.Y., Y.H.J., W.D.X., L.F.L., H.T.Z. and Y.L.W. performed STM measurements with the help of H.-J.G., B.Q.S., S.X.D. and W.A.H. conducted and supervised DFT calculations. L.W.L., S.X.D., M.O., W.D.X., W.A.H., A.H.C.N. and H.-J.G. analyzed the data and wrote the manuscript. H.-J.G. designed and coordinated the project. All authors discussed the results and commented on the manuscript.

\section{Additional information}

Supplementary information accompanies this paper at http://www.nature.com/ scientificreports

Competing financial interests: The authors declare no competing financial interests.

License: This work is licensed under a Creative Commons

Attribution-NonCommercial-NoDerivs 3.0 Unported License. To view a copy of this license, visit http://creativecommons.org/licenses/by-nc-nd/3.0/

How to cite this article: Liu, L. et al. Reversible Spin Control of Individual Magnetic Molecule by Hydrogen Atom Adsorption. Sci. Rep. 3, 1210; DOI:10.1038/srep01210 (2013). 